\title{
ИЕРАРХИЧЕСКАЯ МОДЕЛЬ НЕЛИНЕЙНОЙ ДИНАМИЧЕСКОЙ СИСТЕМЫ
}

\section{HIERARCHICAL MODEL NONLINEAR DYNAMIC SYSTEM}

Yu. Shevnina

Summary. The paper presents the basic principles of building a hierarchical model of a dynamic system of any nature and complexity. When developing the model, the statement was used that any system consists of a hierarchy of controlling and controlled subsystems. The authors provide a mathematical proof of the finiteness and countability of the hierarchical levels of a nonlinear system. The proposed principles for constructing a hierarchical model of a dynamic system of any complexity with the ability to self-organization can be used in the modeling and description of nonlinear systems of various nature, as well as in the development of a generalized mathematical model of their state.

Keywords: nonlinear system, nonlinear dynamics, system modeling, hierarchical structure of systems.

\author{
Шевнина Юлия Сергеевна \\ К.т.н., дочент, Национальный исследовательский \\ университет МИЭТ, Москва, Зеленоград \\ yusm@rambler.ru
}

Аннотация. В работе представлены основные принципы построения иерархической модели динамической системы любой природы и сложности. При разработке модели использовано утверждение, что любая система состоит из иерархии управляющих и управляемых подсистем. Авторами приводится математическое доказательство конечности и счетности иерархических уровней нелинейной системы. Предложенные принципы построения иерархической модели динамической системы любой сложности со способностью к самоорганизации могут быть использованы при моделировании и описании нелинейных систем различной природы, а также при разработке обобщенной математической модели их состояния.

Ключевые слова: нелинейная система, нелинейная динамика, моделирование систем, иерархическая структура систем.

С.П. Капица, И. Пригожин, Г. Хакен, W. Freeman, J. Kelso, P. Grassberger, I. Procaccia и др.,- что делает её особенно актуальной.

\section{Иерархическал структура Аинамической системы} рактеристик в некоторые моменты времени [1]. Закон изменения состояние динамической системы во времени описывается дифференциальными уравнениями различного порядка. Примеры динамических систем можно найти в различных областях: в физике, биологии, химии, экономике, информатике и др. Моделирование нелинейных систем различной сложности, в том числе и со способностью к самоорганизации, является междисциплинарным инструментом исследования разнообразных процессов в природе, технике, обществе $[2,3,4]$. При этом важно разработать единые методологические подходы к представлению и описанию динамических систем. Данные подходы должны позволять на основе обобщенных законов анализировать состояние разнообразных динамических систем. Основная проблема при разработке единых подходов состоит в математическом представлении динамической системы с учетом всех ее характеристик и состояний с приемлемой погрешностью. В последние десятилетия данная задача привлекает все большее число ученых: А.Н. Колмогоров, В.И. Арнольд, Ю.С. Ильяшенко,
Одним из способов решения описанной выше проблемы является построение иерархической структуры нелинейной системы. Этот способ основан на утверждении, что любая система представляет собой иерархию автономных подсистем, в которой исходящие от верхнего уровня команды управления предопределяют переходы подсистем более низких уровней от одного режима функционирования к другому [2].

Существуют два основных подхода к декомпозиции: функциональный и процессный $[4,5,6]$. В первом разделение на подсистемы происходит на основе функций многократно повторяемых действий. Функциональный подход применяется для моделирования регулярной, многократно повторяющейся деятельности. В процессном подходе декомпозиция строится на процессах - устойчивой деятельности, преобразующей входные воздействия в результат. Для нелинейных систем из-за их склонности к самоорганизации и хаотичности 


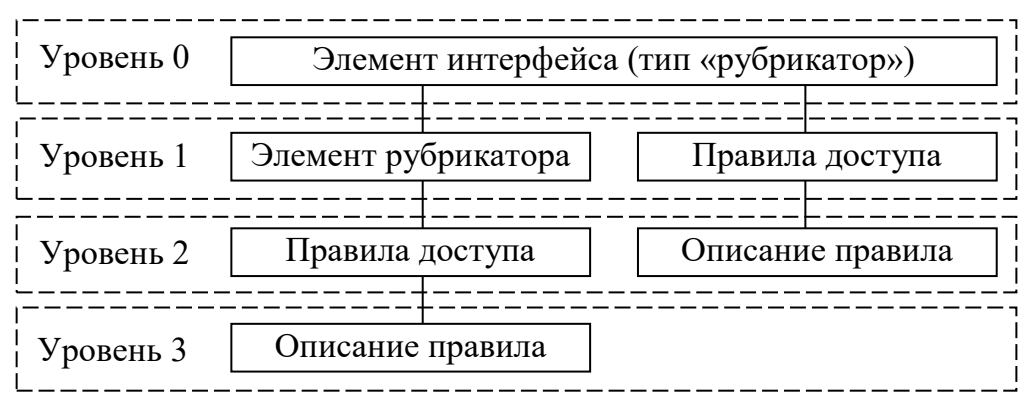

Рис. 1. Представление элемента интерфейса в виде дерева

сложно определить функции, поскольку они не постоянны. В то время как процессы, происходящие в нелинейных системах, постоянны, имеют результат и критерий завершения.

В процессе разделения системы на подсистемы более высоких уровней получаем ее иерархическую модель, а упрощенное графическое представление такой декомпозированной системы назовем иерархической структурой.

Иерархическая структура представляет собой дерево, то есть граф без замкнутых маршрутов с расположением вершин по определенным уровням (рис. 1). Вершина верхнего (нулевого) уровня называется корнем и соответствует исходной системе. Такой подход был использован в [7] при математическом описании процесса формирования профилированных интерфейсов пользователей для доступа к объединенным корпоративным информационным ресурсам.

Для представления иерархической структуры используются деревья разного типа: И-дерево, ИЛИ-дерево, а также И-ИЛИ-дерево. В графе, соответствующему И-дереву, вершины, расположенные на одинаковых уровнях, являются обязательными элементами вышерасположенных подсистем. В ИЛИ-дереве на одинаковых уровнях располагаются вершины возможных элементов подсистем или их варианты. Наиболее часто применяют И-ИЛИ-дерево, в котором уровни с обязательными элементами подсистем соединяются с уровнями вариантов всех или части этих элементов. Сочетание И- и ИЛИ- уровней может быть произвольным.

Количество уровней и вершин иерархической структуры системы может быть произвольно, но обязательно конечно и счетно. Докажем это. Для доказательства используем математическую модель организации доступа к объединенным корпоративным информационным ресурсам [7]. Организация доступа к информационным ресурсам математически пред- ставляет собой нелинейную динамическую систему, поскольку не может быть задана линейным уравнением. Кроме этого, обладает важным свойством нелинейных систем - состоянием, которое зависит от различных внешних факторов (роли пользователя, его разрешений, точки доступа к ресурсам и т.п.). Следовательно, процесс организации доступа является частным случаем описываемой в данной статье сложной динамической системой со способностью к самоорганизации и его математическая модель, построенная с применением иерархических принципов и описывающая изменение состояния системы, может быть использована для доказательства конечности иерархической структуры.

Для доказательства конечности иерархической структуры введем ряд определений [8]. Событие - это выполнение условия добавления подсистемы в иерархическую структуру. Обработчиком события называется блок команд, которые будут выполняться при наступлении события. Правильным называется такой обработчик события, работа которого заканчивается за конечное число шагов. При дальнейшем описании процесса формирования иерархической структуры рассматриваем только правильные обработчики.

Обработка событий при формировании структуры организуется с помощью очереди $Q . Q=\left\{q_{1}, \ldots, q_{n}\right\}-$ множество вызовов обработчиков событий. Если $i<j$, то событие $q_{i}$ обрабатывается раньше события $q_{j}$. Помещение события в очередь означает то, что в очередь помещается вызов обработчика этого события. Обозначим $Q_{t}$ состояние очереди после обработки $t$ первых элементов, если $Q_{t-1} \neq \varnothing . Q_{0}$ - состояние очереди до начала обработки.

События из очереди обрабатываются последовательно и если в процессе исполнения обработчика появляются новые события, то они помещаются в конец очереди. Исполнение одного блока команд приводит к помещению в очередь конечного числа событий. 


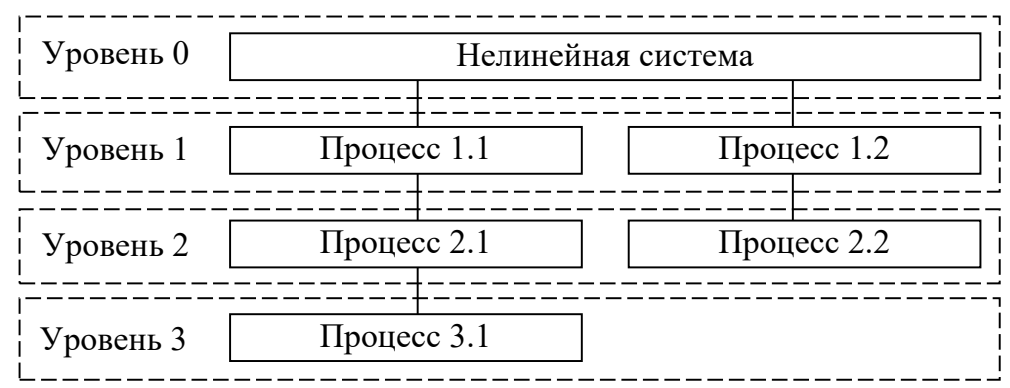

Рис. 2. Представление процессов нелинейной системы в виде дерева

В очередь не попадают одинаковые события, т.е. в одном иерархическом представлении системы не может быть одинаковых элементов.

Описание процессов нелинейной системы конечно и счетно, т.е. для построения иерархической структуры определено конечное и счетное число событий. Поэтому для обработки конкретной системы существует не более чем конечное и счетное множество $E$ всех событийе.

$$
\begin{aligned}
& E=\left\{e_{i}\right\}_{i}^{N} \\
& E-\text { число событий конечное и счетное. }
\end{aligned}
$$

Пусть $Q_{0}=\{q\}$, т.е. в очереди только одно событие $q$ по обработке элементов системы, $M_{1} q-$ события, порождаемые обработкой события $q$. Это может быть декомпозиция подсистемы на ряд более мелких процессов. Если $M_{1} q \neq \varnothing$, то процесс обработки будет продолжаться. $M_{n} q$ - последовательность событий, порождаемых при обработке последовательности $M_{n-1} q$. Если $M_{n-1} q$ пуста, то и $M_{n} q$ пуста.

$$
M_{n+1} q=\bigcup_{\substack{k=1 \\ e_{k} \in M_{n} q}}^{M_{k} q} M_{1} e_{k}, n=1,2
$$

$k$ - порядковый номер события в последователь-ности.

Если в $Q_{0}$ больше одного элемента, то

$$
M_{n+1} Q_{0}=\bigcup_{\substack{k=1 \\ e_{k} \in M_{n} Q_{0}}}^{M_{n} Q_{0}} M_{1} e_{k}, M_{1} Q_{0}=\bigcup_{\substack{k=1 \\ e_{k} \in Q_{0}}}^{Q_{0}} M_{1} e_{k}, n=1,2, \ldots
$$

Обработка событий заканчивается тогда, когда заканчиваются события в очереди (т.е. заканчиваются процессы в системе).

$$
\exists N: M_{N} Q_{0}=\varnothing
$$

Докажем, что число шагов обработки очереди конечно.

$$
\begin{aligned}
& \text { Условие } \\
& \forall t \forall e \in Q_{t} \forall N e \notin M_{N} e
\end{aligned}
$$

при выполнении, которого в очередь не попадают события, порождающие сами себя, т.е. не происходит зацикливаний при выполнении программных блоков.

Для начала изобразим элемент системы в виде дерева (рис. 2). Элементы системы можно представить несколькими типами деревьев. На рис. 2 изображен самый сложный тип дерева И-ИЛИ: элемент 0 уровня состоит из нескольких вариативных процессов. Для краткости представления, на рисунке изображено только два таких процесса. В реальном описании процессов нелинейной системы может присутствовать несколько вариативных процессов у различных процессов, но в любом случае их количество конечное и счетное.

Уровень $i\left(L_{i}\right)$ - это все вершины дерева, построенные на $i$-ом шаге обработки событий формирования иерархической структуры. Вершины дерева соответствуют событиям. Всего таких уровней $n$, где $n-$ число шагов обработки событий по формированию иерархической структуры нелинейной системы.

Шаг 0. На 0 шаге необходимо выбрать одно событие $q$, которое станет корнем дерева $L_{0}=\{q\}$.

Шаг $i$. Для каждой вершины дерева (элемента) $s \in L_{n-1}: M_{1} s \neq \varnothing$ добавить элементы последовательности $M_{1} s$. При $M_{1} s=\varnothing$ дерево будет построено, т.е. структура сформирована.

В качестве примера построим элемент иерархической структуры, соответствующий И-ИЛИ дереву, представленному на рис. 2. Пусть $q$ - событие по обработке 


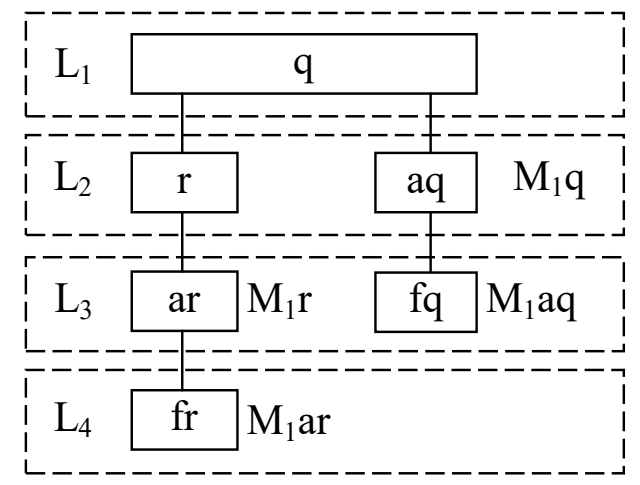

Рис. 3. Элемент иерархической структуры, представленный в виде дерева

разделения подсистемы на основные процессы, $r-$ событие по добавлению Процесса 1.1, aq-событие по добавлению Процесса $1.2, f q$ - событие по добавлению Процесса 2.1, ar - событие по обработке Процесса 2.2 и $f r-$ событие по добавлению Процесса 3.1. Тогда получим:

$M_{1} q=\{r, a q\}, M_{1} r=\{a r\}, M_{1} a q=\{f q\}, M_{1} a r=\{f r\}$, $M_{1} f q=\varnothing, M_{1} f=\varnothing$. Или в виде дерева (рис. 3).

Учитывая условие $\forall t \forall e \in Q_{t} \forall N e \notin M_{N} e$, а также то, что в результате обработки одного события в очередь не попадают одинаковые события, в дереве не может быть более $|E|$ уровней. Также отсюда следует, что для любой вершины $k$ уровня существует не более $|E|-k$ подвершин. Соответственно, в каждом уровне содержится не более чем

$$
p_{k}=\frac{(E-1) !}{(E-k) !}, k=\overline{1,|E|}
$$

элементов, а все дерево состоит не более чем из

$$
V=\sum_{k=1}^{E} p_{k}=\sum_{k=1}^{E} \frac{(E-1) !}{(E-k) !}
$$

вершин. Построенное так дерево соответствует попаданию событий в очередь, очередь обрабатывается не более, чем за $V$ шагов.

Конечность и счетность уровней и вершин иерархической структуры нелинейной системы доказана.

\section{Результаты}

Определим основные принципы построения иерархической структры нелинейных систем.

1. Каждое разделение системы или подсистемы образует свой уровень. Исходная подсистема располагается на нулевом уровне. После ее разделения получаются подсистемы первого уровня. Разделение этих подсистем или некоторых из них приводит к формированию подсистем второго уровня и т.д.

2. Нелинейная система разделяется только по одному, постоянному для все уровней, признаку. Для нелинейных систем в качестве такого признака целесообразно использовать процесс изменения состояния системы, этап жизненного цикла и подобное. При построении И-ИЛИ-деревьев в некоторых случаях возможно сочетание нескольких признаков. Например, при декомпозиции процесса конечного уровня может быть использован функциональный признак.

3. Выделяемые в результате декомпозиции подсистемы должны полностью характеризовать исходную нелинейную систему. При этом, в ИЛИ-дереве выделяемые подсистемы должны полностью взаимно исключать друг друга.

4. Количество выделяемых в процессе декомпозиции нелинейной системы уровней и вершин иерархической структуры определяется требованиями детализации и удобства восприятия получаемой структуры, ее соответствия условиям задачи, в рамках которой происходит данное разбиение, и уровню знаний работающему с ней специалисту. Разбиение системы на подсистемы происходит до такого уровня, на котором понимание устройства подсистем или их описание доступно исполнителю. Таким образом, иерархическая структура всегда субъективно ориентирована: для более квалифицированного специалиста она будет менее подробна.

\section{Зак^ючение}

Предложенные принципы построения иерархической модели динамической системы любой сложности со способностью к самоорганизации могут быть использованы при моделировании и описании нелинейных систем различной природы, а также при разработке обобщенной математической модели их состояния. 


\section{ЛИТЕРАТУРА}

1. Х Хакен Г. Информация и самоорганизация: Макроскопический подход к сложным системам. М.: Мир, 1991

2. Садовский В.Н. Системный подход и общая теория систем: статус, основные проблемы и перспективы развития.— М.: Наука, 1980.

3. Черногор С.А. Введение в синергологию и сложные системы моделирования.—М.: Наука, 2008. — 346 с.

4. Мирошник И.В., Никифоров, В.О., Фрадков А.Л. Нелинейное и адаптивное управление сложными динамическими системами. - СПб.: Наука, 2000. 548 c. - (Сер.: Анализ и синтез нелинейных систем). ISBN5-02-024872-X

5. Тюкин И. Ю., Терехов В.А. Адаптация в нелинейных динамических системах — Санкт-Петербург: ЛКИ, 2008. — 384 с. — (Серия: Синергетика: от прошлого к будущему) — ISBN978-5-382-00487-7

6. Ц Цурков В.И. Декомпозиция в задачах большой размерности. Под ред. Г.С. Поспелова. 1981. 352 с.

7. Шевнина Ю.С. Формирование интерфейсов пользователей для доступа к объединенным корпоративным информационным ресурсам: дис. канд. тех. наук: 05.13.01.- М., 2007. - 174 c.

8. Самарский, А.А. Математические модели. Идеи. Методы. Примеры [Текст] / А.А. Самарский, А.П. Михайлов. — Изд. 2-е, испр. 一 М.: Физматлит, 2002.

9. Пригожин И. От существующего к возникающему: Время и сложность в физических науках. М.: «Наука», 1985.

10. Богданов А.А. Тектология: Всеобщая организационная наука. Редколлегия В.В. Попков (отв. ред.) и др. Сост., предисловие и комментарии Г.Д. Гловели. Послесловие В.В. Попкова.—М.: «Финансы», 2003, С. 287.

11. Tsirlin, A., Gagarina, L. Finite-time thermodynamics in economics // Entropy, 2020, 22(8), 891 https://doi.org/10.3390/e22080891

12. Shevnina, Yu.S., Sokolova, N. Yu., Kyaw Zaw Ye. Organization of Remote Interaction Between Lecturer and Student During Completion of Study Assignment// Proceedings of the 2021 IEEE Conference of Russian Young Researchers in Electrical and Electronic Engineering, EIConRus 2021, cтp. 2076-2080. D0I: 10.1109/ ElConRus51938.2021.9396197

(c) Шевнина Юлия Сергеевна ( yusm@rambler.ru ).

Журнал «Современная наука: актуальные проблемы теории и практики»

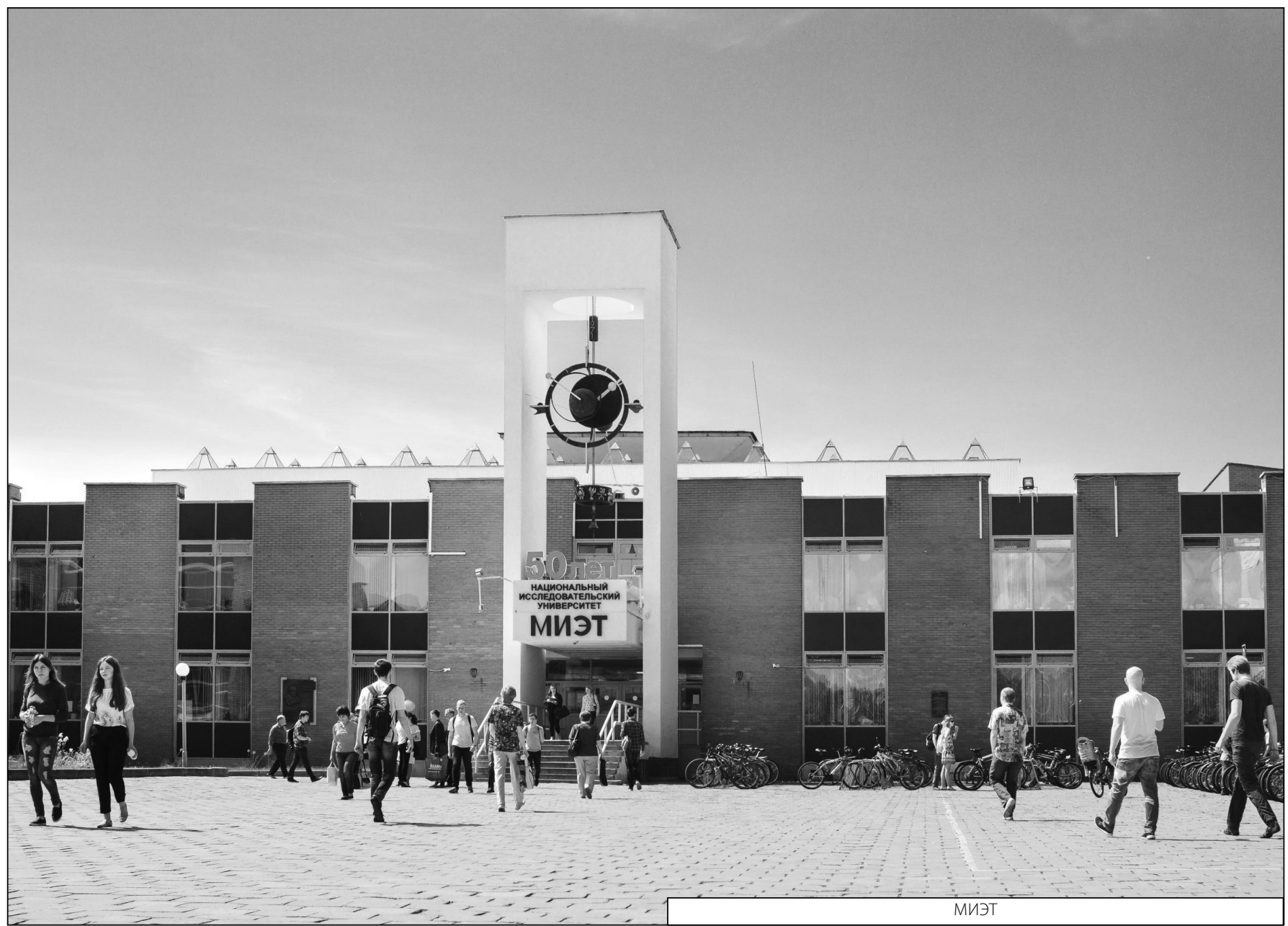

